\title{
Fractal Parametric Oscillator as a Model of a Nonlinear Oscillation System in Natural Mediums
}

\author{
Roman I. Parovik ${ }^{1,2}$ \\ ${ }^{1}$ Institute of Space Physics Research and Radio Wave Propagation, Far East Branch Kamchatskiy kray, Paratunka, Russia \\ ${ }^{2}$ Branch of Far Eastern Federal University, Petropavlovsk-Kamchatsky, Russia \\ Email: romano84@mail.ru
}

Received October 16, 2012; revised December 5, 2012; accepted February 1, 2013

\begin{abstract}
The paper presents a model of fractal parametric oscillator. Showing that the solution of such a model exists and is unique. A study of the solution with the aid of diagrams Stratton-Ince. The regions of instability, which can occur parametric resonance. It is suggested that this solution can be any signal, including acoustic.
\end{abstract}

Keywords: Parametric Resonance; Fractal Analysis; Strutt-Ince Diagram; Plastic Deformation; Fractal Oscillator

\section{Introduction}

It is known that the natural medium (geological medium) may have fractal properties. These properties characterize the spatial-temporal nonlocality or "memory" of the medium, which in turn is determined by the power laws.

Usually geological medium with fractal properties described in terms of fractional calculus [1] by equations with fractional parameters, which depend on the fractal dimension of the geomedium. This fact allows us to make extensive use of mathematical constructions of fractional calculus in a variety of fields, such as the development of new methods of vector-phase acoustic diagnostic of plasticity geological medium [2].

In this paper the nonlinear parametric oscillatory process in the geological medium with fractal properties. Feature of this process is that the displacement of the points geomedium a result of its stress-strain of the state of can occur with increasing amplitude due to changes in the parameters of the medium itself.

Through this process may be described cracks in the avalanche geomedium, which in most cases is preceded by seismic activity in the region (Kamchatka), which can be used in prediction of strong earthquakes.

\section{Statement of the Problem}

The geomedium formed loose deposits of rocks. Assume that this medium has fractal properties. Then the problem of displacement geomedium points in time $t>0$ can be stated as follows:

$$
\partial_{0 t}^{\beta} x(\tau)+\left[\delta+\xi \cos _{\alpha}(\omega t)\right] x(t)=0,
$$

with the initial conditions of the problem

$$
x(0)=C_{1}, x^{\prime}(0)=C_{2} .
$$

Here $x(t)$-shift function geomedium;

$\cos _{\alpha}(\omega t)=E_{\alpha}\left(-(\omega t)^{\alpha}\right)=\sum_{k=0}^{\infty} \frac{(-1)^{k}(\omega t)^{\alpha k}}{\Gamma(\alpha k+1)}$ the generalized cosine with $\alpha$ parameter $1<\alpha<2$. Taking $\alpha=2$ get the usual cosine, i.e., $\cos _{2}(\omega t)=\cos (\omega t)$ [3], $\partial_{0 t}^{\beta} x(\tau)=\frac{1}{\Gamma(2-\beta)} \int_{0}^{t} \frac{x^{\prime}(\tau)}{(t-\tau)^{\beta-1}} \mathrm{~d} \tau$-the fractional differential order $1<\beta<2, \delta$ and $\xi$-parameters of medium. Assume, $\alpha$ and $\beta$ parameters of geomedium, as they are depend on its fractal dimension.

The Equation (1) is a generalization of parametric resonance the classical Mathieu's equation in a case $\alpha=\beta=2$.

Note if put $\alpha=2, \delta=\omega^{\beta}, \xi=0$, then Equation (1) is known as equation of fractional oscillator, which is investigated in study [4].

Since Equation (1) is considered first, then call it a fractal equation parametric oscillator.

\section{Solution}

In study [5] have shown that the solution of equation Cauchy problems (1) and (2) can be represented in the form the Volterra integral equation of the second kind:

$$
x(t)-\int_{0}^{t} K(t-\tau) x(\tau) \mathrm{d} \tau=g(t) .
$$

The kernel of the Equation (3) 


$$
K(t, \tau)=\xi(t-\tau)^{\beta-1} E_{\beta, \beta}\left[-\delta(t-\tau)^{\beta}\right] \cos _{\alpha}(\omega \tau)
$$

and

$$
g(t)=C_{1} E_{\beta, 1}\left(-\delta t^{\beta}\right)+C_{2} t E_{\beta, 2}\left(-\delta t^{\beta}\right) .
$$

If put $\xi=0$ in solution (3), it is the solution of the fractional oscillator

$$
x(t)=C_{1} E_{\beta, 1}\left(-\delta t^{\beta}\right)+C_{2} t E_{\beta, 2}\left(-\delta t^{\beta}\right) .
$$

Solve the Equation (3), use the composite trapezoidal quadrature formula. Take a grid $t_{i}=h(i-1)$ with step $h$. Put $t=t_{i}$ in (3), obtain:

$$
x\left(t_{i}\right)-\int_{0}^{t_{i}} K\left(t_{i}-\tau\right) x(\tau) \mathrm{d} \tau=g\left(t_{i}\right) .
$$

The integral in expression (5) approximate the sum, considering $\tau=t_{j}$, obtain:

$$
\begin{aligned}
& x\left(t_{i}\right)-\sum_{j=1}^{i} A_{i, j} K\left(t_{i}-t_{j}\right) x\left(t_{j}\right)=g\left(t_{i}\right), i=1,2, \cdots, n . \\
& A_{i, 1}=A_{i, i}=\frac{h}{2}, A_{i, 2}=\cdots=A_{i, i-1}, i=2,3, \cdots, n \text {-are co- }
\end{aligned}
$$

efficients of the quadrature formula, $e_{i}$ - the approximation error. Solution (6) can be reduced a system of algebraic equations:

$$
\begin{aligned}
& x_{1}=g_{1}, x_{i}=\frac{g_{i}+h \sum_{j=1}^{i-1} \gamma_{j} K_{i, j} x_{j}}{1-0.5 h K_{i, j}}, \\
& i=2,3, \cdots, n, \gamma_{j}=\left\{\begin{array}{ll}
0.5, & j=1 \\
1, & j>1
\end{array} .\right.
\end{aligned}
$$

The denominator of (7) must satisfy $1-0.5 h K_{i, j} \neq 0$. This condition can be achieved by changing the step $h$. Trapezoidal quadrature formula on the interval $\left[t_{k}, t_{k+1}\right]$ has an error $O\left(h^{3}\right)$, and the total error in the segment$\left[0, t_{k}\right]-O\left(h^{2}\right)$.

The numerical solution of (7) allows the study of fractal parametric oscillator in particular can make the visualization of calculation results.

\section{Numerical Modeling}

Numerical simulation of (1) and (2) was realized using a mathematical software MAPLE. First there was the case when in (1) $\alpha=\beta=2$. It's the classical Mathieu equation. It is known that solution of the Cauchy problem (1)(2), taking $C_{2}=0$ and $\omega=1$ can be written in terms of the Mathieu function. The MAPLE gives the following result:

$$
x(t)=C_{1} \text { Mathieu } C\left(4 \xi,-2 \delta, \frac{1}{2 t}\right)
$$

The solution (8), will used as a test for the analysis of the numerical solution obtained by the method (7). The simulation results for $\alpha=\beta=2$ of the method (7) and formula (8) are shown in Figure 1.

In Figure 1(a) shows that the solution (7) coincides with the solution (8). Amplitude of the oscillations increases Figure 1(b), due to the effect of parametric resonance.

Figure 2 is shown results of simulation when $\alpha=2$, $1<\beta<2, \xi=0.5, \delta=0.02, C_{1}=1 ; C_{2}=0$.

According to this diagram, it's impossible to determine at what values of parameters $A$ and $m$ parametric resonance occurs, for example when $A=m=1$ parametric resonance occurs in Figure 1(b).

In Figure 3 is shown results of simulation when $\alpha=2$, $1<\beta<2, A=0.5, m=0.02, u_{0}=1 ; u_{0}=0$.

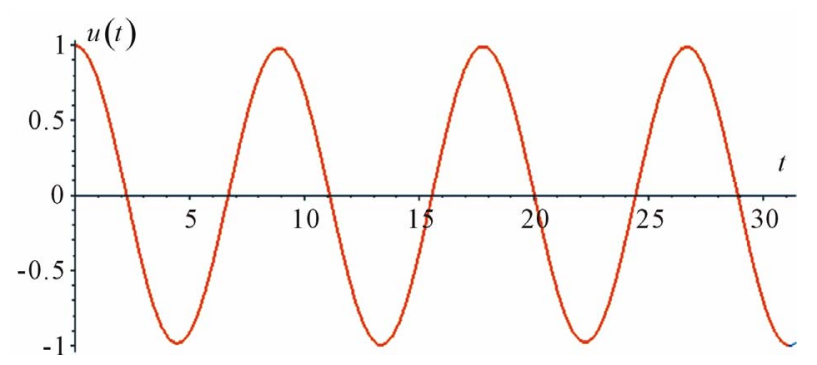

(a)

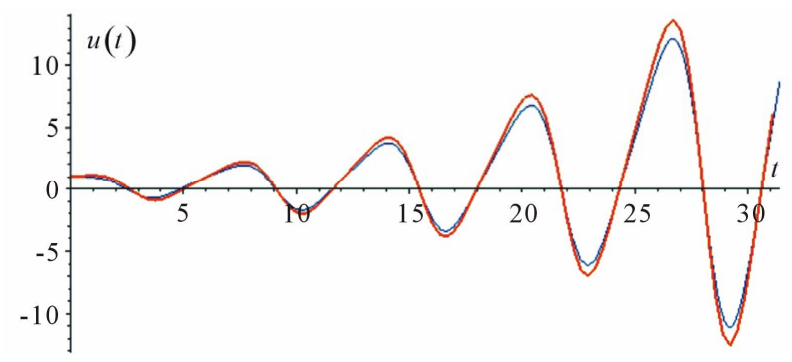

(b)

Figure 1. The calculated curves based on formula (7) (blue curve) and the exact solution (8) (red curve) for the left image parameters: $\xi=0.02, \delta=0.01: C_{1}=1 ; C_{2}=0$, for the right picture settings: $\xi=\delta=1: C_{1}=1 ; C_{2}=0$.

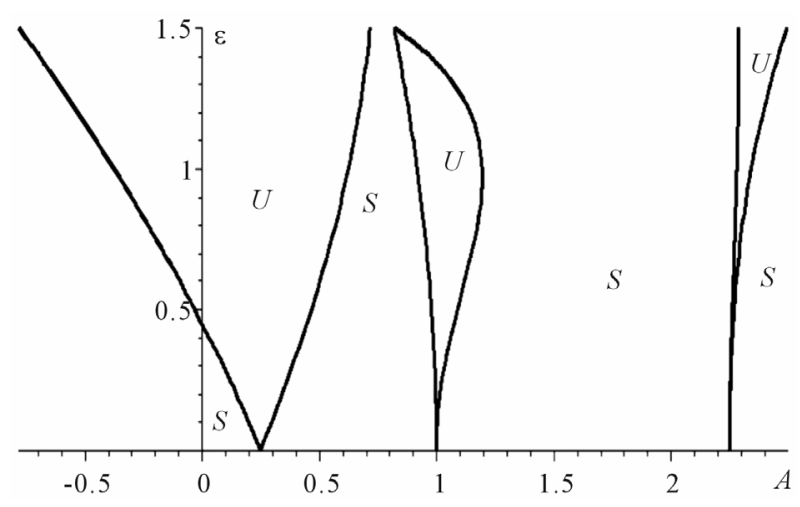

Figure 2. Strutt-Ince diagram stability $(S)$ and instability $(U)$ areas for the classical Mathieu equation. 


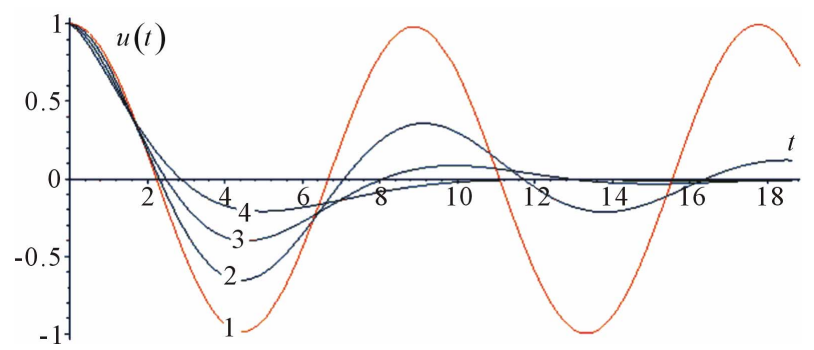

Figure 3. The calculated curves are plotted depending on the values $\beta$ at a fixed value $\alpha=2$, curve $1: \beta=2$, curve $2: \beta$ $=1.8$; curve $3: \beta=1.6$, curve $4: \beta=1.4$.

In this case the solutions haven't a property of periodic, and have a decaying character. These solutions are characteristic of media with dissipation, in particular for inhomogeneous, fractal mediums.

In Figure 4 shows the calculated curves for fixed values of the parameters $\beta=1.8, \xi=0.5, \delta=0.02, C_{1}=1$, $C_{2}=0$ and they are depend of the parameter $\alpha$.

Curves are also damped character at short times behave the same, while at large time intervals is regrouping of curves in the reverse order.

\section{Strutt-Ince Diagram}

Indeed the values of parameters $\xi$ and $\delta$ are entered the so-called zone of instability that can be constructed using of diagrams Strutt-Ince [6].

In Figure 5 is shown a Strutt-Ince diagram stability $(S)$ and instability $(U)$ areas for the classical Mathieu equation.

According to this diagram, it's impossible to determine at what values of parameters $\xi$ and $\delta$ parametric resonance occurs, for example when $\xi=\delta=1$ parametric resonance occurs in Figure 1(b).

Consider the differential Equation (1) and fractional derivatives for $\alpha=2$ and $\omega=1$ :

$$
\partial_{0 t}^{\beta} x(\tau)+[\delta+\xi \cos (t)] x(t)=0
$$

Define the conditions under which there is a parametric resonance in (9). To do this, in the $\delta-\xi$ plane must construct diagrams Strutt-Ince. As a rule, there is a region of instability, parametric resonance, which leads to an increase in the amplitude of oscillations

Usually in the area of instability exists parametric resonance, which leads to an increase in amplitude. Estimate the parameters $\delta$.

Consider the derivative of fractional order on the left side of (9):

$$
\begin{aligned}
\partial_{0 t}^{\beta} x(\tau) & =\frac{1}{\Gamma(2-\beta)} \int_{0}^{t} \frac{x^{\prime \prime}(\tau) \mathrm{d} \tau}{(t-\tau)^{\beta-1}} \\
& =\frac{1}{\Gamma(2-\beta)} \int_{0}^{t} v^{1-\beta} x^{\prime \prime}(t-v) \mathrm{d} v
\end{aligned}
$$

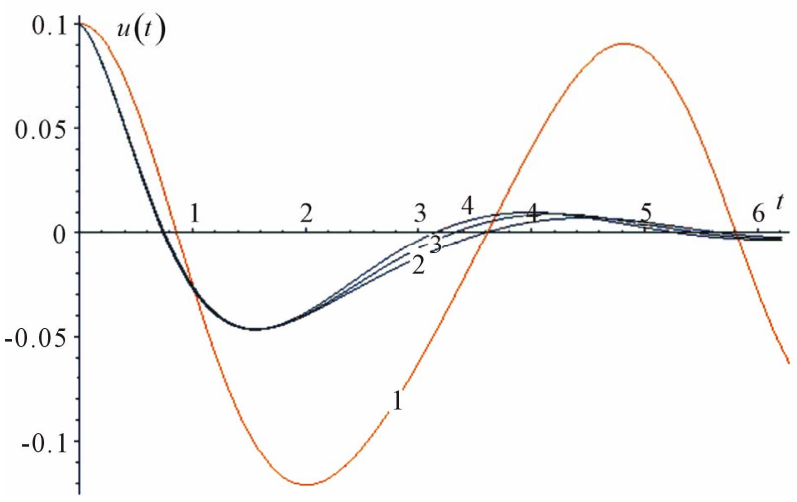

Figure 4. The calculated curves plotted according of the parameter values $\alpha$ for a fixed value $\beta=1.8: \alpha=2$-curve 1, $\alpha=1.8$-curve $2, \alpha=1.6$-curve $3, \alpha=1.4-$ curve 4 .

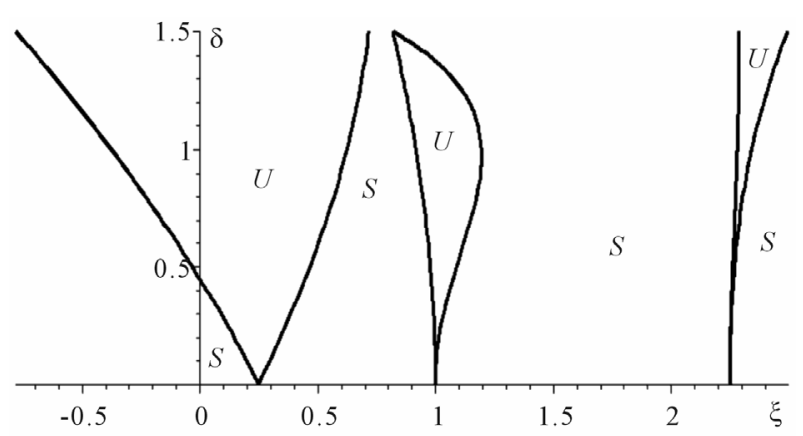

Figure 5. Strutt-Ince diagram for the mathieu equation. The letter $U$ indicated the region of instability, when possible parametric resonance, $S$ - the region of stability.

Use the method of harmonic balance for Equation (9), its solution formed of a harmonic series [7]:

$$
x(t)=\sum_{n=0}^{\infty} A_{n} \cos \frac{t n}{2}+B_{n} \sin \frac{t n}{2}
$$

For the first resonance take the first harmonic (11), i.e. $n=1$ and substitute (9) in view of the representation (10). After some transformations go to the following result:

$$
\begin{aligned}
\delta= & \frac{1}{2^{\beta}} \sin ((\beta-1) \pi / 2) \\
& \pm \frac{1}{2^{\beta+1}} \sqrt{2^{2 \beta} \xi^{2}-4 \cos ^{2}((\beta-1) \pi / 2)}
\end{aligned}
$$

If in (12) to put $\beta=2$, get the known relation for the first classical parametric resonance Mathieu

$$
\delta=\frac{1}{4} \pm \frac{\xi}{2}
$$

In Figure 6, as an example, is built Strutt-Ince diagram according to the expression (13).

It can be noted that in (12) imposes constraints on the parameters $\xi$. The value must satisfy the following inequality: 


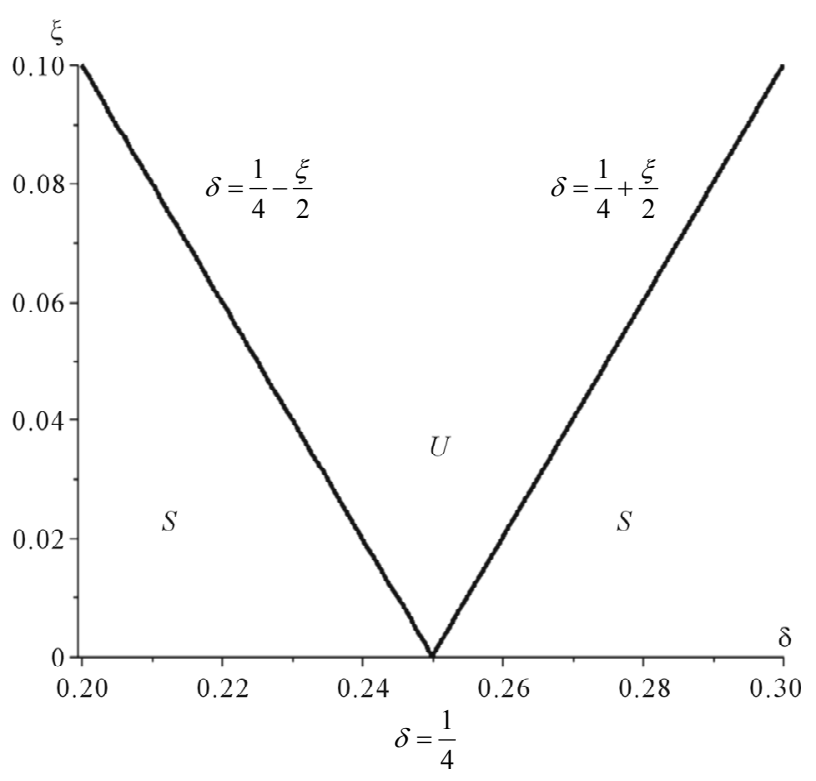

Figure 6. Strutt-Ince diagram for expression (13).

$$
\xi=\frac{\cos ((\beta-1) \pi / 2)}{2^{\beta-1}}
$$

Figure 7 shows the area of parameter values $\xi$ according to (14).

Spend the visualization of the results of research solutions of (9). According to the above analysis, it was the expression (12). Below is its visualization:

Figure 8 shows that a decrease in the parameter $\beta$ changes of the curves, i.e., change the boundaries of the stability and instability. Instability area is narrowed for the values of $\beta \rightarrow 1$, so the effect of parametric resonance is reduced.

Figure 9 shows the surface constructed according to (12), depending on the parameters $\beta, \delta, \xi$. There is an area on the surface, where the values of parameter $\delta$ is not defined, it is caused by the expression (14).

Analysis of the solution of Equation (1) shows that when the parameter $\beta$ narrows the field instability, also the parameter $\delta$ has restrictions (14).

The boundaries of the stability and instability in the Strutt-Ince diagram can be improved if consider the solution (11) for the harmonics $n>1$, but it will lead to some computational difficulties.

\section{Conclusions}

The paper presents a model of fractal parametric oscillator. This model generalizes previously known models: the classical oscillator $(\xi=0, \alpha=\beta=2)$, a parametric oscillator $(\alpha=\beta=2)$ and fractal oscillator $\left(\alpha=2, \delta=\omega^{\beta}\right.$, $\xi=0)$.

The solutions of the Cauchy problem (1.2) using numerical methods (7), showed a good agreement of the

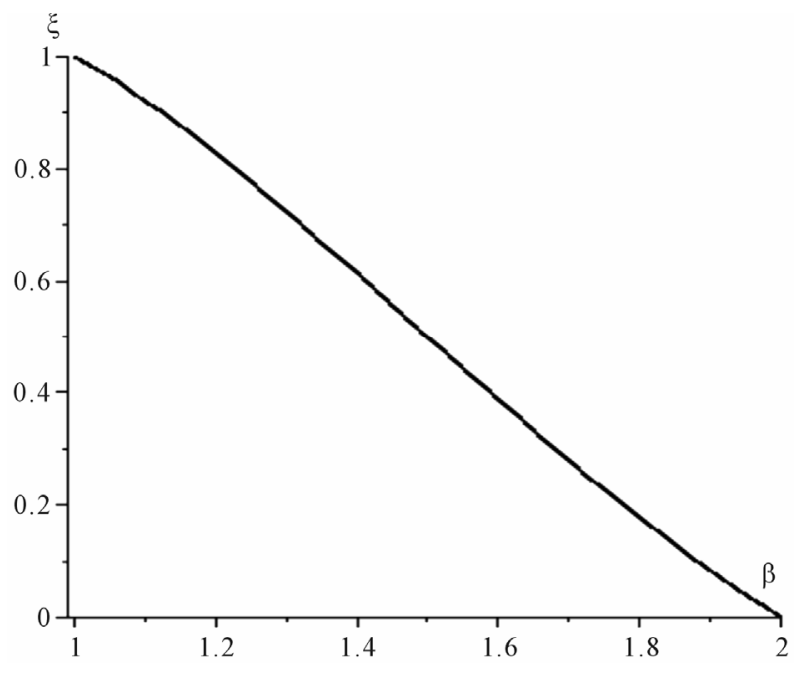

Figure 7. Curve defining limits at the parameter $\xi$.

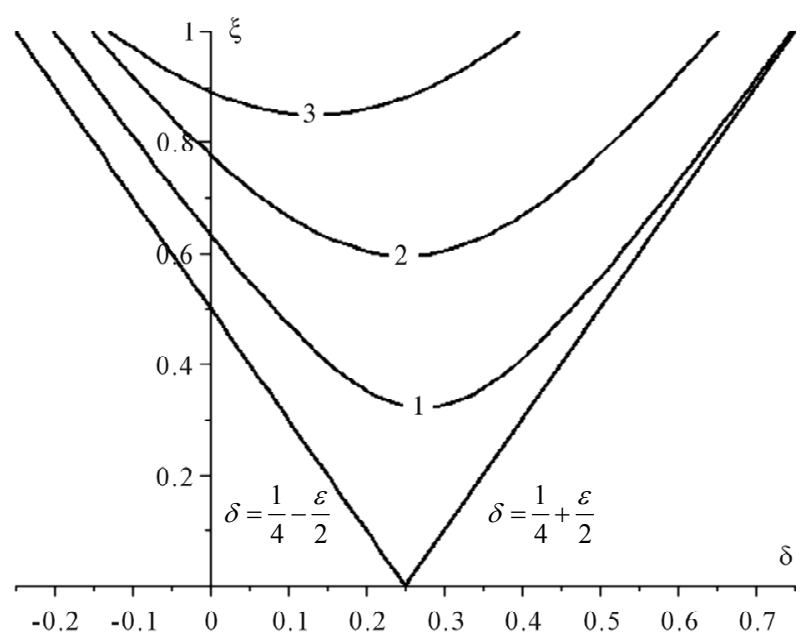

Figure 8. Ince-Strutt diagram for expression (12). Curves are plotted a function of the parameter on parametr $\beta$ and $\alpha$ $=2$ : 1) $\beta=1.8$; 2) $\beta=1.6$; 3) $\beta=1.2$.

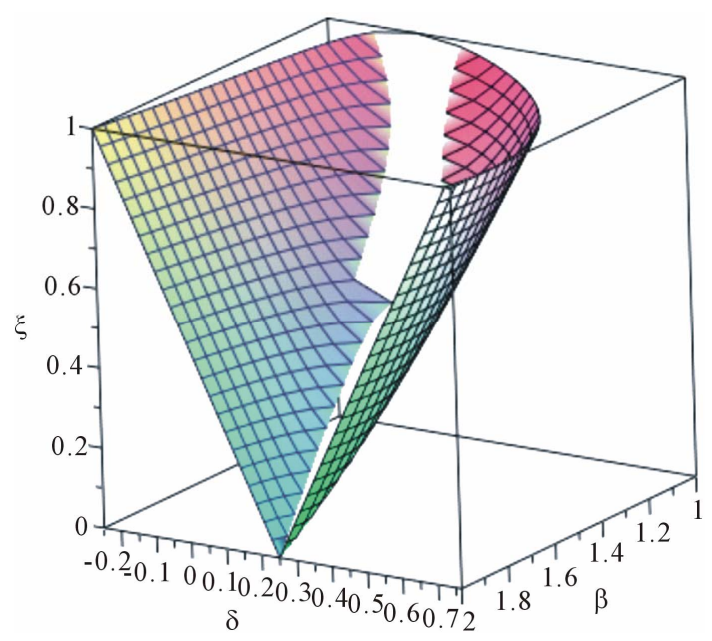

Figure 9. $\alpha-\delta-\xi$ surface constructed according expression (12). 
numerical solution of (7) with the exact solution (8).

Explore the first parametric resonance for Equation (9) with the harmonic balance method (11). With Strutt-Ince diagrams identify areas of instability, the existence of parametric resonance. It is shown that the values $\beta \rightarrow 1$ of these areas are narrow. Therefore the probability that the parameters $\xi$ and $\delta$ in such areas is reduced.

Further interest is the study of the Equation (9)—calculation of the second or the third resonance, as well as the more general Equation (1). There is interest in the study of the phase trajectories for the solution (3).

There is interest in the equation with the right side. It should be noted that in study [8], the authors considered the equation of the fractional oscillator with an external stabilizing inertial effects. It would be interesting to consider the Equation (1) subject to an external force, according to this work, and then proceed to the consideration of (1) with a random external force.

\section{Acknowledgements}

The work was performed as part of the program DPS RAS IV.10 "Fundamentals of acoustic diagnostics of artificial and natural environments" and is supported by RFBR (grant no. 11-01-90715).

\section{REFERENCES}

[1] A. M. Nakhushev, "Fractional Calculus and Its Application," Fizmatlit, Moscow, 2003, p. 272.

[2] V. A. Gordeenko, "Vector-Phase Methods in Acoustics," Fizmatlit, Moscow, 2007, p. 480.

[3] V. A. Nakhusheva, "Differential Equations of Mathematical Models of Non-Local Processes," Nauka, Moscow, 2006, p. 173.

[4] R. P. Meilanov and M. S. Yanpolov, "Features of the Phase Trajectory of a Fractal Oscillator," Technical Physics Letters, Vol. 28, No. 1, 2002, pp. 67-73. doi:10.1134/1.1448634

[5] R. I. Parovik, "Cauchy Problem for Non Local Mathieu Equation," Doklady AMAN, Vol. 13, No. 2, 2011, pp. 90-98.

[6] F. Van de Pol and M. J. Strutt, "On the Stability of the Solutions of Mathieu's Equation," Philosophical Magazine, Vol. 5, 1928, pp. 18-38.

[7] R. H. Rand, S. M. Sah and M. K. Suchrsky, "Fractional Mathieu Equation," Communications in Nonlinear Science and Numerical Simulation, Vol. 15, 2010, pp. 3254 3262 .

[8] V. V. Afanas'ev and M. J. E. Daniel, "Polish Stabilization of the Inertial Effects of the Fractal Oscillator," Technical Physics Letters, Vol. 36, No. 7, 2010, pp. 1-6. 